\title{
5 Perspektiven für die Soziologie des Nichtwissens
}

Diese Studie hat gezeigt, dass die in Diskursen um neue Technologien entstehende Fokusverschiebung von Wissen auf Nichtwissen zu einem Lenkungsdispositiv geführt hat. Im Kontext von Nichtwissen und dem damit verknüpften Lenkungsdispositiv entstehen in Gesellschaften der reflexiven Modernisierung drei zentrale Strategien. Wie diese Studie gezeigt hat, betreffen diese das Abschätzen, das Regulieren und das partizipative Einbinden von Anspruchsgruppen und der Zivilgesellschaft in den Diskurs. Damit einher gehen einerseits übergreifend wahrnehmbare gesellschaftspolitische Veränderungen und andererseits entstehen länderspezifisch unterschiedliche kulturelle Ausprägungen des Lenkungsdispositivs.

Die übergreifenden gesellschaftspolitischen Veränderungen der Fokusverschiebung von Wissen auf Nichtwissen manifestieren sich allgemein 1) in veränderten Konstellationen der involvierten Akteure, 2) in neu gesetzten thematischen Schwerpunkten und 3) in neuartigen Praktiken des Abschätzens, des Regulierens und des Einbindens von Anspruchsgruppen und zivilgesellschaftlichen Akteuren in Nichtwissensfragen. Wie diese Studie am Fall des mit der Nanotechnologie verbundenen Nichtwissens gezeigt hat, lassen sich dabei drei zentrale Phänomene erkennen:

1) die gesellschaftliche Distribution von Expertise

2) die Verschmelzung von entscheidungsrelevanten Wissensformen

3) die Auflösung von institutionenspezifischen Handlungspraktiken

Diese in Nichtwissensdiskursen übergreifend wahrnehmbaren Phänomene werden im nachfolgenden Kapitel 5.1 ausgeführt. Daneben zeigt diese Studie aber auch unterschiedliche kulturelle Ausprägungen im Lenken von Nichtwissen in den analysierten nationalen, internationalen und supranationalen Kontexten. Diese unterschiedlichen Lenkungskulturen, die trotz ihrer lokalen Divergenzen zu weitgehend vergleichbaren Nanotechnologiepolitiken geführt haben, werden im Kapitel 5.2 diskutiert. Diese erstaunliche Konvergenz der Ergebnisse von divergenten soziokulturell geprägten politischen Herangehensweisen untermauern die im Kapitel 5.3 präsentierten Schlussfolgerungen. Diese zeigen, wie in Nichtwissensdiskursen feststellbare Phänomene und neue Konstellationen; nämlich die gesellschaftliche Distribution von Expertise, die Verschmelzung von ent- 
scheidungsrelevanten Wissensformen und die Auflösung von institutionsspezifischen Handlungspraktiken zu einem Zustand maximaler Gestaltungsoffenheit und minimaler Verbindlichkeit führen. Statt den erwünschten Klärungen im Sinne einer robusten Technologiepolitik tragen die im Rahmen des Lenkungsdispositivs zum Nichtwissen ergriffenen Aktivitäten nicht zur Reduktion von Nichtwissen, sondern vielmehr zu dessen Erhöhung bei. Dabei nehmen in diesen neuen Konstellationen Unklarheiten zu. So wird zunehmend unklar, wer Handlungs- und Durchsetzungskompetent ist und wer nicht. Dadurch läuft das Lenkungsdispositiv in Leere und das Nichtwissen wird nicht reduziert, sondern sogar erhöht. Neben dem mit der Nanotechnologie einhergehenden Nichtwissen entsteht zusätzliches Nichtwissen, nämlich solches bezüglich der wissenschafts- und technikpolitischen Zuständigkeit. Dabei wird zunehmend unklar, wer in technikpolitischen Fragen kompetent ist, wer die Entscheidungen bezüglich ihrer Regulierung fällt und wer die damit verbundene gesellschaftspolitische Verantwortung für neue Technologien und ihre Implikationen trägt.

Selbst das Argument, dass die Auflösung eindeutiger Wissensautoritäten und autorisierter Institutionen doch einen Erfolg des Dispositivs darstellt, trifft hier nicht zu. Zwar führt diese Auflösung dazu, dass die Nanotechnologie maximal gestaltungsoffen agieren kann und nur minimalen verbindlichen regulatorischen Restriktionen ausgesetzt ist. Fälschlicherweise wird dieser Zustand maximaler Gestaltungsoffenheit und minimaler Verbindlichkeit als Voraussetzung für Innovationsprozesse gesehen. Wie die vorliegende Analyse jedoch gezeigt hat, werden gerade in Nichtwissensdiskursen verbindliche regulatorische Richtlinien als innovationsfördernd wahrgenommen (vgl. Kapitel (3.2.2).

\subsection{Gesellschaftliche Phänomene in Nichtwissensdiskursen}

Die Analyse des Nanotechnologiediskurses in Europa und in den Vereinigten Staaten hat gezeigt, dass die Zuständigkeit sowohl über die inhaltliche Ausgestaltung als auch über die potenziellen Nebenfolgen neuer Technologien durch das in Nichtwissensdiskursen auftretende Lenkungsdispositiv zunehmend an die Gesamtgesellschaft übertragen wird. Diese Studie bestätigt die von Böschen und Wehling (2012, 324f.) für Nichtwissensdiskurse beschriebene Auflösung der traditionellen Arbeitsteilung zwischen Wissenschaft und Politik, worin erstere für das ,entscheidungsrelevante Wissen' und letztere für dessen Einbindung in Entscheidungsprozesse zu- 
ständig sind. Vielmehr sind neu nun sowohl Wissenschaft als auch Politik und — wie diese Studie zeigt — auch weitere gesellschaftliche Anspruchsgruppen wie die Industrie und Umwelt- und weitere Schutzorganisationen in die Produktion von entscheidungsrelevantem Wissen als auch in die damit verbundenen Entscheidungsprozesse eingebunden.

Die von Böschen and Wehling (2004, 10ff.) für Nichtwissensdiskurse beschriebene Auflösung vermeintlich stabiler Grundunterscheidungen, wie diejenige zwischen ,Experten' und ,Laien', ,Fakten' und ,Werten' und zwischen ,wissenschaftlichem' und ,nichtwissenschaftlichem' Wissen, lässt sich auch im politischen Diskurs der Nanotechnologie nachweisen. Im Abschätzungsdiskurs verschwimmt die Unterscheidung zwischen Experten und Laien nicht nur bei der Analyse potenzieller Technikfolgen, sondern auch bei der inhaltlichen Bestimmung der Nanotechnologie. Hier sind neben den Vertretern der wissenschaftlichen Fachgemeinschaft (z.B. der Ingenieurswissenschaften, der Physik, Chemie und Biologie), der wissenschaftlichen Standesorganisationen (z.B. der britischen Royal Society, der Royal Academy of Engineering und der Europäischen Akademie) auch solche aus Wissenschaftspolitik, Politik und Verwaltung (z.B. der US-amerikanischen NSF, der Europäischen Kommission, des britischen EPSRC und der deutschen DFG) und aus der Technikfolgenabschätzung (z.B. aus dem OTA, dem TAB und dem ITAS Karlsruhe) ebenso wie Futuristen (z.B. Eric Drexlers Foresight Institute) involviert. Damit wird die Expertise, wie auch Lösch (Lösch 2014, 190) feststellt, nicht nur durch konkurrierende Wissensformen, sondern auch durch die divergenten Interessenslagen der als Experten auftretenden Akteure geprägt, was Weingart (2001, 159) als ,Inflationierung der Expertise' bezeichnet.

Sowohl in die Abschätzung der potenziellen Folgen der Nanotechnologie als auch in ihre Regulierung sind neben den genannten Vertretern der Wissenschaft, der Standesorganisationen und der Technikfolgenabschätzung auch solche aus weiteren natur-, sozial- und kulturwissenschaftlichen Forschungsbereichen (wie Toxikologie, Umwelt-, Gesundheits- und Sicherheitsforschung aber auch aus Rechts-, Sozialwissenschaften und Ethik), aus Politik und Verwaltung (z.B. Umwelt-, Arbeitsschutz-, und Gesundheitsbehörden und deren Beratungsgremien), aus der Industrie und der Dienstleistungsbranche (z.B. Hersteller/chemische Industrie, Branchenverbände, Rückversicherung, Dienstleister) aus angewandten Forschungs- und Beratungsorganisationen (z.B. dem Wilson-Zentrum, dem Meridian Institut, Demos und dem Ökoinstitut) und solche aus Umweltund Konsumentenschutzorganisationen (wie z.B. der ETC Gruppe, En- 
vironmental Defense Fund, Greenpeace, Friends of the Earth und WHICH?) einbezogen. Diese gesellschaftliche Distribution von Expertise zeigt sich auch im Partizipationsdiskurs. Durch die verstärkte Einbindung der unterschiedlichen Anspruchsgruppen wird deren Wissen auch für Entscheidungsträger verfügbar gemacht. Dies gilt insbesondere für die Anspruchsgruppenpartizipation. Wie die Analyse der Ansätze der zivilgesellschaftlichen Partizipation gezeigt hat, fehlen jedoch die Ideen, wie zivilgesellschaftliches Wissen in den politischen Prozess übersetzt werden kann. Wie auch Lösch $(2014,190)$ feststellt, führen die wissensproduzierenden Verfahren der Selbstregulierung der Nanotechnologie weder zu gesichertem Wissen noch zu einem Konsens, an dem sich politische Entscheidungsträger orientieren könnten. In den unterschiedlichen Dialogverfahren werden keine Entscheidungen gefällt, sondern vielmehr vielfältige Einschätzungen, Bewertungen und Vorschläge gesammelt, die weder ,empirisch rekonstruierbar' noch ,legitimatorisch bedeutsam' sind (vgl. ibd.).

Expertise wird also gesellschaftlich distribuiert. Die inhaltliche Expertise wird in Fragen nach dem Potenzial einer neuen Technologie, ihren Folgen und ihrer Regulierung in unterschiedlichen gesellschaftlichen Bereichen und unter Anwendung unterschiedlicher Praktiken generiert. Solche Expertise generierenden gesellschaftlichen Bereiche umfassen vielfältigste Akteure, wie solche aus der Wissenschaft (wie beispielsweise aus der Forschung, aus Fachgemeinschaften und Standesorganisationen), aus der Wirtschaft (wie beispielsweise aus Industrie- und Dienstleistungsunternehmen und Branchenverbänden), aus der Politik (wie beispielsweise aus den Behörden, dem Parlament und aus anderen politischen Organisationen und Institutionen), aus Nichtregierungsorganisationen (wie beispielweise aus dem Umwelt- und Konsumentenschutz, aus privaten Stiftungen, Denkfabriken und aus forschungsnahen Organisationen).

Die vorliegende Analyse deutet zudem darauf hin, dass sich die vielfältigen Expertisen des Abschätzungs-, des Regulierungs- und des Partizipationsdiskurses verweben und situativ unabhängig ihrer Herkunft in politische Programme aufgenommen werden. Im Abschätzungsdiskurs sind beispielsweise Erkenntnisse aus dem Nanotechnologieprojekt am WilsonZentrum in den politischen Diskurs in den Vereinigten Staaten eingeflossen. Mitarbeiter des Wilson-Zentrums haben ihre Expertise in den Kongress und in verschiedene kritische Analysen eingebracht, wie z.B. diejenigen des NRC (siehe Kapitel 2.3.7). In Großbritannien haben die kritischen Analysen von Greenpeace und DEMOS die Nanotechnologiepolitik der Regierung ebenso mitgeprägt wie die wissenschaftlichen Studien des 
Forschungsrates für Ingenieurs- und Physikwissenschaften (EPSRC). Diese unterschiedlichen Einschätzungen haben dazu geführt, dass die britische Regierung die Royal Society und die Royal Academy of Engineering mit einer Technik- und Implikationsanalyse beauftragt hat.

Im Regulierungsdiskurs führt die beschriebene Auflösung der traditionellen Arbeitsteilung gesellschaftlicher Bereiche, wie der Wissenschaft und der Politik und insbesondere der Politik und der Wirtschaft zu weiteren Konsequenzen. Die traditionelle Funktionsteilung, dass die Politik für die Regulierung zuständig und die Wirtschaft von dieser betroffen ist, wird aufgelöst. Dies geschieht durch das Entwickeln selbstregulatorischer Ansätze durch die verschiedenen Unternehmen und privatwirtschaftlichen Stiftungen und Organisationen. Anders gesagt wird durch die Bedeutung, welche die industrielle Selbstregulierung im Nanotechnologiediskurs einnimmt, die Funktionsteilung zwischen der Politik und der Wirtschaft aufgehoben. Damit nehmen insbesondere Hersteller, die sich selbst regulieren, eine Doppelfunktion ein; nämlich diejenige der Regulierenden und der von der Regulierung betroffenen.

Prägend für den Partizipationsdiskurs sind die insbesondere in Großbritannien und in Deutschland neu eingesetzten Anspruchsgruppendialoggremien wie die deutsche NanoKommission und das britische Nanotechnologie Anspruchsgruppen Forum (NSF). Hier fällt auf, dass trotz der in Abschätzungsberichten omnipräsenten Empfehlung nach zivilgesellschaftlicher Partizipation, der Bevölkerung kaum Expertenstatus zugewiesen wird. Bis auf den partizipativen Ansatz des EPSRC, indem Vertreterinnen und Vertreter der Zivilgesellschaft die Festlegung von Forschungsschwerpunkten mitgeprägt haben, spielt der politische Einfluss der zivilgesellschaftlichen Expertise im Nanotechnologiediskurs selbst beim prominent propagierten britischen ,upstream engagement' kaum eine Rolle.

Die Analyse des Nanotechnologiediskurses zeigt eine Fülle an unterschiedlichen thematischen Ebenen, auf welchen die Nanotechnologie inhaltlich definiert und ihre potenziellen Nebenfolgen verhandelt werden. Insbesondere in ihrer inhaltlichen Rahmung in den Nationalen Förderprogrammen wird hier über Böschen und Wehlings (2004, 10ff.) Feststellung des Verschmelzens von ,Fakten' und ,Werten' hinausgehend von einem weitgehenden Bedeutungsverlust wissenschaftlich produzierten Wissens 
gegenüber spekulativem Wissen ausgegangen. ${ }^{164}$ Die übergreifende inhaltliche Rahmung der Nanotechnologie erfolgt in nationalen Förderprogrammen, insbesondere in den Vereinigten Staaten, hauptsächlich auf der Basis von Visionen, Spekulationen und Utopien. Dabei wird die Lösung drängender zivilisatorischer Fragen in den Bereichen Gesundheit, Umweltschutz, Ernährungssicherheit und die Verbesserung der menschlichen Leistung in Aussicht gestellt. Im Rahmen der US-amerikanischen nationalen Nanotechnologieinitiative werden umfassende nanotechnologische Visionen als realistische Zukunftsszenarien kommuniziert. Damit einhergehend hat der Verweis von Nanotechnologieforschenden auf revolutionäre Verbesserungen nahezu aller Lebensbereiche durch die Nanotechnologie in den Vereinigten Staaten dazu geführt, dass die Politik von der Notwendigkeit der Lancierung eines nationalen Nanotechnologieprogrammes überzeugt worden ist. In den anderen untersuchten Ländern und auf Unionsebenen scheint die Publikation einer nationalen Nanotechnologiestrategie von einem gewissen Nachahmungseffekt geprägt zu sein. Insbesondere Deutschland lehnt sich auch inhaltlich an der US-amerikanischen Innovationsrhetorik an und prägt die Nanotechnologie in verschiedenen visionären Kontexten, wie in dem der, ökologischen Modernisierung'.

Wie die vielfältigen Schwierigkeiten gezeigt haben, sich auf eine einheitliche formale Definition zu einigen, die über die Beobachtung einer veränderten Reaktivität im Nanogrößenbereich hinausgeht, lässt sich die Nanotechnologie inhaltlich kaum auf eine abgeschlossene technologische Entwicklung zurückführen. Diese in den Vereinigten Staaten initiierte übergreifende Definition der Nanotechnologie basierend auf Visionen, hat auch den europäischen Diskurs und die Nanotechnologiestrategien insbesondere Deutschlands aber auch diejenige der Europäische Union und die Förderungsschwerpunkte in Großbritannien geprägt. Im Diskurs über die inhaltliche Rahmung der Nanotechnologie spielt also Wissen, das im Kontext von akademischer Forschung produziert wird, eine vergleichbare,

164 In der sozialwissenschaftlichen Wissenschafts- und Technikforschung wird die Kategorisierung von Wissen in ,Fakten' und ,Werte' verschiedentlich problematisiert (z.B. Latour 1987; Latour 1995). Dabei besteht auch hier die Überzeugung, dass ,wissenschaftlich produziertes Wissen' und ,spekulatives Wissen' nicht trennscharf voneinander abgegrenzt werden können (vgl. dazu auch Maasen \& Weingart 2000). Für das Argument dieses Kapitels gilt eine Diskussion dieses Unterschieds dennoch als wesentlich, so dass der Widerspruch wissentlich in Kauf genommen wird. 
wenn nicht sogar eine untergeordnete Rolle zu spekulativem Wissen. Relevant wird wissenschaftlich hergestelltes Wissen jedoch dann, wenn es um formale oder technische Definitionen geht. Dies geschieht auf der Ebene von Disziplinen und Forschungsprojekten und in der Analyse von gesundheitlichen, umwelt- und sicherheitsrelevanten Nebenfolgen sowie mehrheitlich auch in der Analyse von ethischen, rechtlichen und sozialen Implikationen.

Neben der substanziellen inhaltlichen Rahmung der Nanotechnologie in nationalen Förderinitiativen - insbesondere in denjenigen der Vereinigten Staaten und Deutschlands - auf von wissenschaftlichen Erkenntnissen losgelösten Visionen, hat spekulatives Wissen auch den Diskurs um potenzielle Nebenfolgen geprägt. Dabei haben Dystopien wie das grey goo Szenario, das in Michael Crichtons Roman Prey, vom britischen Thronfolger, in der Debatte zwischen Eric Drexler und Richard Smalley und in Berichten der Umweltorganisation ETC aufgenommen worden ist, die wissenschaftspolitische Behandlung der Nanotechnologie in allen untersuchten Ländern beeinflusst. In Großbritannien und in Deutschland hat insbesondere der Diskurs um die Dystopien zu verschiedenen Abschätzungsstudien geführt, wie diejenigen der Royal Society und der Royal Academy of Engineering und des Büros für Technikfolgenabschätzung des deutschen Bundestags. Auch in den Vereinigten Staaten haben Dystopien den politischen Diskurs beeinflusst. Sie haben einerseits Initiativen von regierungsexternen Organisationen wie diejenige des Wilson-Zentrums zur Erarbeitung eines Nanotechnologieprojektes (PEN) und die des Zentrums für biologische und umweltrelevante Nanotechnologie (CBEN) an der Rice Universität zur Etablierung eines internationalen Nanotechnologierates (ICON) begünstigt. Andererseits haben die verstärkten Diskussionen um potenzielle Nebenfolgen auch zur Einrichtung von ELSI-Forschungsschwerpunkten im Rahmen der Nationalen Nanotechnologieinitiative und zur verstärkten Finanzierung von EHS-Forschung geführt. Auf der Ebene der Europäischen Union haben insbesondere die im Royal Society Bericht prominent aufgegriffenen Diskussionen über Nebenfolgen die Rahmung der Nanotechnologiestrategie hinsichtlich des Einschlusses von potenziellen Implikationen beeinflusst.

Auch im Abschätzungsdiskurs hat sich gezeigt, dass sowohl wissenschaftlich produziertes Wissen als auch spekulatives Wissen eingesetzt wird. Dies betrifft insbesondere die Fokusverschiebung von ELSI zu EHS. Während unter ELSI häufig spekulatives Wissen formuliert worden ist, spielen in EHS toxikologische Studien in der Verhandlung der potenziel- 
len Nebenfolgen eine wichtige Rolle. Im Regulierungsdiskurs haben die in diesen toxikologischen Studien produzierten wissenschaftlichen Erkentnisse über nachteilige Gesundheitseffekte von Nanomaterialien zwar zu verschiedenen freiwilligen und selbstregulatorischen Regulierungsmaßnahmen geführt, nicht aber zu einer über einzelne Produkte hinausgehenden gesetzlichen Regulierung.

Wie die analysierten Schlussberichte aus partizipativen Gremien und Veranstaltungen zeigen, haben hier Erkenntnisse aus nach traditionellen wissenschaftlichen Methoden aufgebauten Studien, die beispielsweise Hypothesen prüfen und quantitativ nachweisbare Effekte zeigen, eine ebenso bedeutsame Rolle gespielt wie außerwissenschaftlich produziertes und spekulatives Wissen. Außerwissenschaftliches Wissen wird im Nanotechnologiediskurs von verschiedenen Anspruchsgruppen produziert und entsteht in unterschiedlichen gesellschaftlichen Konstellationen, wie in der behördenübergreifenden Zusammenarbeit oder in der Kooperation zwischen unterschiedlichen Anspruchsgruppen. Dabei wird es auch nicht alleine in klassischen TA-Studien oder Expertisen, wie der Royal Society Studie, publiziert, sondern in vielfältigen Formen, Formaten und Texten, wie Pamphleten, Blogs, Websites, und Auftragsstudien.

Auch im Regulierungsdiskurs zeigt sich der Bedeutungsverlust von wissenschaftlich produziertem Wissen. Das Vorsorgeprinzip wird darauf angelegt, dass selbst bei der Abwesenheit von wissenschaftlich gesicherten Daten regulatorische Schritte ergriffen werden können. Trotz des Vorliegens von wissenschaftlichen Studien, die von der Zelltoxizität von Nanomaterialien ausgehen, hat die europäische - dem Vorsorgeprinzip unterliegende Regulierungskultur — nicht dazu geführt, dass auf Unionsebene oder in den untersuchten Mitgliedsstaaten über einzelne Produkte hinausgehende gesetzliche Maßnahmen ergriffen werden. Vielmehr werden die Erkenntnisse aus den toxikologischen Studien als noch nicht ausreichender Beweis einer Gefährdung angesehen. Dabei haben sie sowohl in den Vereinigten Staaten als auch in Europa zu freiwilligen Regulierungsansätzen, wie dem britischen und dem US-amerikanischen freiwilligen Datenmeldeverfahren und zu einzelnen, produktspezifischen gesetzlichen Maßnahmen geführt. Weiter zeigt sich im Regulierungsdiskurs die politische Erweiterung der Entscheidungsfindung über Regulierungsfragen hinaus. Der Zusammenschluss der europäischen Staaten zur Union und der Bedeutungsgewinn der internationalen Koordination, wie dies in den OECD-Gremien erfolgt, erweitert die Behandlung von Regulierungsfragen 
neben der nationalen auch um die supranationale und die internationale Ebene.

In Nichtwissensdiskursen ist also die Unterscheidung von Fakten und Werten, von gesichertem bzw. verlässlichem Wissen und spekulativem Wissen kaum mehr möglich. Unterschiedliches Wissen findet Eingang in den Diskurs um die Nanotechnologie und wird von verschiedenen Entscheidungsträgern aufgenommen. Mit der gesellschaftlichen Distribution von Expertise verteilt sich die Produktion von entscheidungsrelevantem Wissen unter den verschiedenen gesellschaftlichen Anspruchsgruppen.

Diese Studie hat gezeigt, dass nicht nur die Akteure, die als Experten im Diskurs auftreten und die im Diskurs verhandelten Themen und Wissensinhalte - auf welchen die Entscheidungsträger ihr Handeln abstützen aus den unterschiedlichsten gesellschaftlichen Bereichen stammen. Vielmehr verweben sich in Nichtwissensdiskursen auch die Herkünfte der unterschiedlichen Praktiken. Das heißt, die in den verschiedenen Institutionen traditionell ausgeübten Praktiken, wie — plakativ ausgedrückt — dass die Wissenschaft forscht, die Behörden regulieren, die Firmen produzieren und die Nichtregierungsorganisationen gesellschaftspolitische Themen kritisch reflektieren, werden in Nichtwissensdiskursen aufgelöst. In Nichtwissensdiskursen lassen sich also bestimmte soziale Praktiken nicht mehr zwingend bestimmten gesellschaftlichen Institutionen zuordnen. Vielmehr werden solche von den verschiedenen gesellschaftlichen Bereichen situativ und kontextabhängig übernommen, kombiniert und angewendet. Anders gesagt oder - wiederum plakativ ausgedrückt — produzieren Wissenschaftler auch spekulatives Wissen, Behörden initiieren auch Forschungsstudien, Firmen und Nichtregierungsorganisationen beginnen auch $\mathrm{zu}$ regulieren und Umwelt- und Konsumentenschutzorganisationen auch wissenschaftlich $\mathrm{zu}$ arbeiten. ${ }^{165}$ Es wird hier also ein Verschmelzen der Grenzen nicht nur zwischen wissenschaftlicher und nichtwissenschaftlicher Wissensproduktion (Böschen \& Wehling 2004, 10ff.) beobachtet,

165 Beispiele hierfür sind Richard Denison, ein Vertreter der US-amerikanischen Umweltorganisation Environmental Defense, dessen Berichte auch in der Wissenschaft fachliche Anerkennung finden (Denison 2007), Alexander Arnall von der Universität Reading, der neben seiner wissenschaftlichen Arbeit auch eine Studie für Greenpeace geschrieben hat und James Wilsdon, der vor seiner Professur an der Universität Sussex im Rahmen seiner Anstellung im britischen ThinkThank Demos Berichte für eine verstärkte gleichberechtigte Einbindung der Zivilgesellschaft in wissenschafts- und technikbezogene Entscheidungsfindung verfasst hat. 
sondern grundsätzlich zwischen den von bestimmten gesellschaftlichen Anspruchsgruppen traditionell ausgeübten epistemischen Praktiken und solchen externer Anspruchsgruppen.

Dies zeigt, dass das mit sich neu entwickelnden Spitzenforschungsfeldern und den sich an naturwissenschaftlichen Konvergenzzonen interdisziplinär herausbildenden großtechnischen Entwicklungen verbundene Nichtwissen zu einer gesellschaftlichen Distribution von Expertise führt. Ebenso führt es zum Verschmelzen der Grenzen zwischen unterschiedlichen Wissensformen, wobei spekulatives Wissen ebenso wie akademisch produziertes Wissen in die Entscheidungsfindung miteinfließt. Ebenso werden gesellschaftliche Praktiken wie die akademische Wissensproduktion, die Regulierung und Dissidenz nicht mehr alleine in ihren angestammten Institutionen praktiziert. Vielmehr werden sie situativ und kontextabhängig von den jeweils unterschiedlichen Anspruchsgruppen aufgegriffen und übernommen. In der Entscheidungsfindung über technologische Entwicklungen etablieren sich also neue Formen der Wissensproduktion und des politischen Handelns ebenso wie neuartige institutionelle und personelle Konstellationen. Diese führen — wie in Kapitel 5.3 gezeigt wird - jedoch nicht wie mit dem Lenkungsdispositiv intendiert zu mehr Wissen oder zur Auflösung des Nichtwissens. Vielmehr führen diese zu dessen Erhöhung. Damit verlieren die zuständigen gesellschaftlichen Instanzen ihre traditionellen Kompetenzen in der Entscheidungsfindung und in der Verantwortungsübernahme in wissenschaftspolitischen Fragen. Dies führt zu einem grundsätzlichen Verlust in der wissenschaftspolitischen Lenkung von neuen Technologien.

Die in diesem Kapitel übergreifend dargestellten, in Nichtwissensdiskursen feststellbaren gesellschaftlichen Phänomene und Verschiebungen zeigen sich in den untersuchten nationalen, internationalen und supranationalen Kontexten jeweils in unterschiedlichen kulturellen Ausprägungen. Wie das nachfolgende Kapitel zeigt, lassen sich dabei vier verschiedene Lenkungskulturen unterscheiden. Dass diese trotz ihrer Divergenz zu relativ konvergenten Nanotechnologiepolitiken geführt haben, deutet darauf hin, dass die in Nichtwissensdiskursen feststellbaren Phänomene und neuen Konstellationen, nämlich die gesellschaftliche Distribution von Expertise, die Verschmelzung von entscheidungsrelevanten Wissensformen und die Auflösung von institutionsspezifischen Handlungspraktiken zu einem Zustand maximaler Gestaltungsoffenheit und minimaler Verbindlichkeit führen, die selbst nationale und kulturelle Eigenheiten überlagern. 


\subsection{Innovation, Integration, Implikation und Konsens: 4 Kulturen im} Umgang mit Nichtwissen

Diese Studie hat gezeigt, dass sich Nichtwissensdiskurse durch die gesellschaftliche Distribution von Expertise, die Verschmelzung der Grenzen zwischen entscheidungsrelevanten Wissensformen und durch die Auflösung von institutionenspezifischen Handlungspraktiken auszeichnen. Trotz dieser kulturraumübergreifenden Gemeinsamkeiten, lassen sich auch länderspezifische Eigenheiten im Umgang mit dem mit Nanotechnologie verbundenen Nichtwissen feststellen. Diese Eigenheiten werden hier als Lenkungskulturen bezeichnet. Diese äußern sich in graduell abweichenden Schwerpunktsetzungen und Konstellationen der involvierten Akteure, der durch sie behandelten Themen und der von ihnen ausgeübten Praktiken in der Abschätzung und der Regulierung der Nanotechnologie und in der pertizipativen Einbindung von Anspruchsgruppen und der Zivilgesellschaft. Dabei wird nachfolgend gezeigt, wie im Umgang mit Nichtwissen in den Vereinigten Staaten eine innovationsbasierte Lenkungskultur entstanden ist. Die Lenkungskultur auf der Ebene der Europäischen Union wird als integrativ bezeichnet, diejenige in Großbritannien als implikationsbasiert und die in Deutschland als konsensorientiert.

Dies mag auf den ersten Blick allzu plakativ und ein wenig simplifizierend erscheinen. Dennoch wird hier die Auffassung vertreten, dass die Qualität einer derart detailreichen Analyse gerade auch in ihrer Zusammenführung der übergreifenden gesellschaftspolitischen und kulturellen Tendenzen liegt. Diese etwas plakative Leseart der in den vorangegangenen Kapiteln dargelegten Erkenntnisse soll hier als heuristisch wertvolle Typologisierung im Stile der idealtypischen Vorgehensweise Max Webers, dazu beitragen, mittels theoretischer Zuspitzung Typen herauszukristallisieren, für die sich zwar in der empirischen Wirklichkeit keine reinen Entsprechungen finden lassen, die aber für die weitere empirische Forschung ein wichtiges Referenzsystem bilden können.

\subsubsection{Vereinigte Staaten: Innovation}

Die Analyse des Nanotechnologiediskurses in den Vereinigten Staaten zeigt eine dominante inhaltliche Prägung der Thematik im Kontext von Innovationen. Dabei steht die Lösung drängender zivilisatorischer Probleme im Vordergrund, die durch eine machtvolle Kooperation zwischen den die 
Nanotechnologie befürwortenden Ingenieur- und Naturwissenschaften und der Politik vorangetrieben wird. Dies geschieht im Kontext der die USamerikanische Wissenschaftspolitik prägenden Haltung der wissensbasierten Ökonomie, die besagt, dass die ausreichende Bereitstellung von Forschungsmitteln die Grundlage des wirtschaftlichen Wohlstandes darstellt. Dem übergeordneten Ziel, eine wissensbasierte Vormachtstellung im internationalen Wettbewerb um Innovation und Marktführerschaft zu erzielen und zu erhalten, werden Bedenken gegenüber potenziell nachteiligen Implikationen untergeordnet und anfänglich selbst soziale Implikationen in nutzbringende gesellschaftliche Anwendungen umgedeutet.

In diesem Sinn wird dem mit der Nanotechnologie einhergehenden Nichtwissen so begegnet, dass das hohe Innovationspotenzial der Technologie prominent betont und potenzielle nachteilige Implikationen möglichst ignoriert oder in Innovationen umgedeutet werden. In diesem Kontext lässt sich auch die regulatorische Zurückhaltung der Regierung erklären und die weitgehende Abwesenheit von partizipativen Verfahren. Die verschiedenen, meist von regierungsexternen Akteuren ergriffenen Maßnahmen mit Fokus auf Implikationen, wie das EDF-DuPont NanoRisk Framework, das PEN-Projekt des Wilson-Zentrums, das ICON der Rice Universität und die Etablierung eines freiwilligen Datenmeldeverfahrens durch die Umweltbehörde zeigen, dass in den Vereinigten Staaten einzelne Akteure wie Behörden, Hersteller, Umweltorganisationen und private Stiftungen und Zentren durchaus vorsorgeorientierte Ansätze initiieren. Jedoch erscheint ihre Wirkung angesichts des machtvollen wissenschaftspolitischen Zusammenschlusses von Politik, Wissenschaft und Wirtschaft und deren prominente Rahmung der Nanotechnologie im Kontext hochrangiger Innovationen als begrenzt. Deren gemeinsame inhaltliche Prägung der Nanotechnologie im Sinne der wissensbasierten Ökonomie fußt auf der Idee, dass durch den starken Fokus auf die Innovationen potenzielle nachteilige Implikationen vernachlässigbar oder im Vergleich zum umfassenden Nutzen der Technologie marginal sind. Deshalb wird die den US-amerikanischen Nanotechnologiediskurs im Umgang mit Nichtwissen dominierende Prägung hier als innovationsbasierte Lenkungskultur bezeichnet. 


\subsubsection{Europäische Union: Integration}

Die Analyse des Umgangs mit dem mit Nanotechnologie einhergehenden Nichtwissen auf Unionsebene hat im Abschätzungsdiskurs eine starke Gegenwartsorientierung und einen Fokus auf Regulierungsfragen gezeigt. Sowohl in der Strategie als auch im Aktionsplan dominiert der Schwerpunkt auf Anwendungen der Nanotechnologie, die sich in absehbarer Zeit realisieren lassen und auf ihre Implikationen. Dabei spielen die traditionellen Institutionen wie die Kommission und das Parlament tragende Rollen im Diskurs. Anders als in den untersuchten Mitgliedsstaaten werden auf Unionsebene kaum nanotechnologiespezifische Gremien eingesetzt und es schalten sich auch kaum regierungsexterne Akteure in den Diskurs ein. Vielmehr erscheint die auf der Unionsebene beobachtete Behandlung der Nanotechnologie in den existierenden Strukturen als Versuch, das mit der Nanotechnologie einhergehende Nichtwissen in die bestehenden politischen Netzwerke, Gremien und Institutionen zu integrieren.

Dabei agieren die zuständigen Generaldirektionen nicht individuell, sondern kommunizieren einheitlich und übergreifend als Kommission in der formalisierten Form der offiziellen Mitteilung. Im Regulierungsdiskurs spielt die divergierende Einschätzung von Kommission und Parlament bezüglich der Frage, ob es sich bei der Nanomaterialienregulierung primär um ein Regulierungs- oder ein Implementierungsproblem handelt, eine zentrale Rolle. Auch dieser Diskurs läuft auf offiziellen und formalisierten Kommunikationswegen ab, die Praktiken wie diejenige der ,Mitteilung' und der ,Resolution' umfassen. Die Frage nach der Partizipation, also der Einbindung von Anspruchsgruppen und der Zivilgesellschaft wird auf der Unionsebene ebenfalls integrativ gelöst. Statt wie in Großbritannien oder in Deutschland, partizipative Gremien und Gefäße neu zu etablieren, wird die Partizipation mit Ausnahme des ,Safety for Success' Dialogs als Förderbereich ins Rahmenprogramm für die Forschungsförderung auf Unionsebene integriert. Durch die Delegation der Partizipation an die bestehenden Forschungsgefäße und ihre Etablierung als wissenschaftliche Konferenz wird die Partizipation auf Unionsebene integriert und verwissenschaftlicht.

Auf der Unionsebene nehmen also im Abschätzungs-, im Regulierungsund im Partizipationsdiskurs um das mit der Nanotechnologie einhergehende Nichtwissen insbesondere traditionelle Institutionen eine zentrale Rolle ein. Sie agieren mittels im politischen Diskurs etablierten offiziellen und formalisierten Kommunikations- und Handlungspraktiken, wie der 
,Mitteilung', der ,Resolution' und der Forschungsförderung. Deshalb wird die den Nanotechnologiediskurs auf Unionsebene prägende Lenkungskultur als integrativ bezeichnet.

\subsubsection{Großbritannien: Implikationen}

Die Analyse des Nanotechnologiediskurses in Großbritannien und des dort praktizierten Umgangs mit Nichtwissen zeigt seit seinem Beginn um die Jahrtausendwende einen starken Fokus auf die Implikationen. Geprägt durch vorangegangene Technikkontroversen um gentechnisch veränderte Organismen und BSE, scheint sich in Großbritannien die Haltung etabliert zu haben, dass sich eine Technologie nur dann entwickeln kann, wenn ihre potenziellen Implikationen im Vorfeld ihrer Förderung von einer gesamtgesellschaftlich anerkannten Autorität - in diesem Fall einem Konsortium bestehend aus der Royal Society und der Royal Academy of Engineering - analysiert und mit den involvierten Akteuren und der Zivilgesellschaft verhandelt worden sind.

Dabei erscheint die Rolle der Royal Society im wissenschaftspolitischen Diskurs Großbritanniens als besonders bemerkenswert. Als wissenschaftspolitisches Beratungsgremium fließen ihre Empfehlungen traditionellerweise direkt in die politische Umsetzung ein. Dabei scheint sie die britische Wissenschaftspolitik über die letzten Jahrzehnte hinweg kontinuierlich und signifikant mitbestimmt zu haben. Beispielsweise ist PUS im Anschluss an ihren Bericht aus dem Jahr 1985, indem sie zum ,public understanding of science' geraten hat, zur offiziellen wissenschaftspolitischen Leitstrategie Großbritanniens geworden. Ebenso hat ihre Empfehlung im Jahr 2005 nach einer verstärkt ,upstream'-orientierten zivilgesellschaftlichen Einbindung im Nanotechnologiediskurs dazu geführt, dass die Partizipation zum neuen wissenschaftspolitischen Paradigma Großbritanniens erklärt worden ist. Damit hat die Royal Society in Großbritannien einen Partizipationsboom ausgelöst, der in keinem der anderen untersuchten Lännder in diesem Umfang beobachtet worden ist. Eine derart einflussreiche wissenschaftspolitische Institution, die als nahezu unhinterfragte Autorität die wissenschaftspolitische Stoßrichtung der britischen Regierung über Jahrzehnte entscheidend prägt, findet sich weder in den anderen untersuchten Nationen noch auf Unionsebene. Dies erscheint gerade in Nichtwissensdiskursen und im Kontext der gesellschaftlichen Distribution von Expertise und dem Autoritätsverlust gesellschaftlicher Insti- 
tutionen in den zeitgenössischen Wissensgesellschaften als augenfällig. Wie die Interviews gezeigt haben, gilt die Royal Society als eine der Institutionen, die nicht nur den britischen, sondern auch den europäischen Nanotechnologiediskurs zentral geprägt hat.

Der in Großbritannien prominent ausgebildete Fokus auf die Implikationen, das Einsetzen von Fachgremien und die vielfältigen Versuche, die Zivilgesellschaft einzubinden, lassen sich zu einem wesentlichen Teil ebenfalls auf die Empfehlungen der Royal Society zurückführen. Dabei fällt die hohe Zahl an nanotechnologiespezifischen Beratungsgremien auf, die im Abschätzungs-, im Regulierungs- und im Partizipationsdiskurs Großbritanniens eingesetzt worden sind. Ebenfalls spezifisch für den britischen Nanotechnologiediskurs gilt die starke Fokussierung auf die zivilgesellschaftliche Einbindung in die wissenschafts- und technikbezogene Entscheidungsfindung. Dabei scheint sich in Großbritannien im Umgang mit dem mit der Nanotechnologie verbundenen Nichtwissen die Überzeugung etabliert zu haben, dass sich die Technologie nur dann robust entwickeln kann, wenn die Implikationen von der Gesamtgesellschaft akzeptiert sind. Dieser dominante Fokus auf die Implikationen und auf die zivilgesellschaftliche Einbindung steht in einem auffälligen Gegensatz zu der USamerikanischen Haltung, die besagt, dass angesichts der überwältigenden Innovationen die Implikationen vernachlässigbar sind. Deshalb wird der britische Umgang mit dem mit der Nanotechnologie verbundenen Nichtwissen hier als implikationsbasierte Lenkungskultur bezeichnet.

\subsubsection{Deutschland: Konsens}

Die Analyse des deutschen Nanotechnologiediskurses zeigt die Bedeutung, welche die Anspruchsgruppenpartizipation und damit einhergehend die Idee, potenzielle Nutzen und Implikationen einer Technologie konsensuell auszuhandeln, einnimmt. Im nationalen Anspruchsgruppendialoggremium der NanoKommission soll die klare Abgrenzung, die im Rahmen der deutschen Nanotechnologiestrategie zwischen der Forschungsförderung im Geiste der wissensbadierten Ökonomie und der Abschätzung von potenziell nachteiligen Implikationen etabliert worden ist, überwunden werden. Dabei fokussieren die deutschen Strategiedokumente zur Nanotechnologie hauptsächlich auf die Forschungsförderung, die in der Kompetenz des Forschungs- und Bildungsministeriums liegt. Demgegenüber ist die Abschätzung der potenziellen Implikationen anfänglich der dafür 
traditionell institutionalisierten Technikfolgenabschätzung und den Vorsorgebehörden übertragen worden.

Mit der NanoKommission wird ein Gremium eingesetzt, in dem mögliche Anwendungen im Kontext einer ökologischen Modernisierung gleichzeitig mit potenziellen Implikationen diskutiert werden. Dabei dominiert die Vorstellung, dass zwischen den beteiligten Akteuren und Anspruchsgruppen im Hinblick auf den Umgang mit der Nanotechnologie ein gesellschaftlicher Konsens erzielt werden sollte.

Diese kooperative Haltung zeigt sich auch im Umgang der verschiedenen Vorsorgebehörden mit dem mit der Nanotechnologie verbundenen Nichtwissen im Abschätzungs- und im Regulierungsdiskurs. Sie verfassen gemeinsame Forschungsstrategien und engagieren sich in Anspruchsgruppendialoggremien. Spezifisch für den deutschen Diskurs ist die aktive Rolle, welche die Herstellerindustrie in dieser Konsenspolitik spielt. Die Hersteller etablieren eigene partizipative Foren, engagieren sich in den bestehenden Gremien und entwickeln auch in Kooperation mit der Arbeitssicherheitsbehörde selbstregulatorische Ansätze.

Der in Deutschland beobachtbare Ansatz im Umgang mit dem mit Nanotechnologie verbundenen Nichtwissen deutet also auf eine starke Orientierung an kooperativen Ansätzen und an der gesellschaftlichen Konsensfindung hin. Die deutsche Nanotechnologiepolitik basiert also auf der Haltung, dass sich die Technologie nur dann robust entwickeln kann, wenn ein gesellschaftlicher Konsens über deren potenziellen Innovationen und Implikationen erzielt wird. Dies wird hier als konsensuelle Lenkungskultur bezeichnet.

\subsubsection{Vier Lenkungskulturen im Umgang mit Nichtwissen}

Die vorangegangenen Kapitel haben länderspezifische Eigenheiten im Umgang mit dem mit der Nanotechnologie verbundenen Nichtwissen aufgezeigt, woraus hier vier verschiedene Lenkungskulturen abgeleitet worden sind. Die im Rahmen dieser Arbeit geführten Interviews deuten darauf hin, dass die unterschiedlichen Lenkungskulturen im Kontext einerseits der gesellschaftspolitischen Historie im jeweiligen Kulturraum und andererseits der vorangegangenen Technikkontroversen zu lesen sind. Die USamerikanische innovationsbasierte Lenkungskultur ist in dieser Auffassung ein Abbild der im Rahmen der wissensbasierten Ökonomie generierten gesellschaftlichen Erwartung an die Wissenschaft und die Politik, um 
drängende zivilisatorische Probleme zu lösen und den Wohlstand zu sichern. Im Kontext eines verblassten Umweltbewusstseins in der US-amerikanischen Bevölkerung (Kapitel 3.2) und des Fehlens unmittelbar vorangegangener Technikkontroversen, wie z.B. in der Gentechnik, werden die Innovationen als zentral und die Implikationen als vernachlässigbar angesehen.

Die integrative Lenkungskultur auf der Ebene der Europäischen Union scheint ein Abbild der Haltung zu sein, dass sich das supranationale Konstrukt der Europäische Union nur dann aufrechterhalten lässt, wenn seine Diversität in formale Strukturen integriert wird bzw. wenn die neugeschaffenen Institutionen eindeutige Rollen und Verantwortlichkeiten haben. Diese Strukturen umfassen bezüglich ihrer Aufgaben und Kompetenzen klar festgelegte Institutionen und offizielle Kommunikationswege. Auch im Nanotechnologiediskurs lässt sich beobachten, dass diese politisch verankerten und vorgegebenen Kommunikations- und Handlungswege eingehalten werden. Dabei lässt sich feststellen, dass, wo immer dies möglich ist, versucht wird, neue Aspekte wie hier die Nanotechnologie und die damit verbundenen Nichtwissensfragen, in den bestehenden institutionellen, gesetzlichen und kommunikativen Rahmen zu integrieren.

Sowohl die implikationsorientierte Lenkungskultur Großbritanniens als auch die konsensuelle Deutschlands lässt sich auf die vorangegangenen Technikkontroversen zurückführen. Die Gentechnik- und in Großbritannien auch die BSE-Kontroverse haben die wissenschaftspolitische Haltung der beiden Länder entscheidend geprägt. In beiden Ländern scheint die Überzeugung gewachsen zu sein, dass sich solche Kontroversen durch die Thematisierung der potenziellen Implikationen und die gesellschaftliche Konsensfindung vermeiden lassen. Dabei liegt der britische Fokus bei der wissenschaftspolitischen Fokussierung auf den Implikationen und der Integration der Zivilgesellschaft und der Anspruchsgruppen in die Entscheidungsfindung. Demgegenüber liegt der Schwerpunkt in Deutschland auf der gesellschaftlichen Konsensfindung im Rahmen von Anspruchsgruppenpartizipation.

Interessanterweise haben die verschiedenen Lenkungskulturen jedoch kaum dazu geführt, dass sich in den untersuchten Ländern eine signifikant unterschiedliche politische Behandlung der Nanotechnologie entwickelt hat. Obwohl die Wege dazu graduell abweichend begangen werden, zeigen sich die Ergebnisse erstaunlich homogen. Alle untersuchten Länder treiben Forschung und Entwicklung im Nanotechnologiebereich unter substanzieller Forschungsförderung entschieden voran. Ebenso lässt sich in 
allen untersuchten Ländern der Vorwurf nachweisen, dass sie die Implikationsforschung und hierin insbesondere den Bereich Umwelt-, Gesundheits- und Sicherheitsforschung unterdurchschnittlich fördern. Dies ist selbst in der hauptsächlich auf die Implikationen fokussierenden Politik Großbritanniens der Fall. Keiner der untersuchten Kulturräume hat die Nanotechnologie gesetzlich umfassend geregelt. Sowohl in der präventivorientierten US-amerikanischen Regulierungskultur als auch im vorsorgeorientierten Ansatz Europas werden maßgebliche Regulierungslücken festgestellt.

Vor diesem Hintergrund stellt sich die Frage, wie diese länderspezifisch unterschiedlichen Lenkungskulturen in insgesamt einheitliche Politiken münden können. Dazu wird hier die Haltung vertreten, dass die in Nichtwissensdiskursen feststellbaren Phänomene und neuen Konfigurationen kulturelle Divergenzen überlagern. Die gesellschaftliche Distribution von Expertise, die Verschmelzung von entscheidungsrelevanten Wissensformen und die Auflösung von institutionsspezifischen Handlungspraktiken haben zu einem transnationalen Zustand der maximalen Gestaltungsoffenheit und der minimalen Verbindlichkeit geführt. Das damit verknüpfte Nichtwissen überlagert also selbst länderabhängig divergierende Lenkungskulturen und trägt dazu bei, dass diese in eine gestaltungsoffene, unbestimmte und übergreifend konvergente Nanotechnologiepolitik verschmelzen.

\subsection{Die Nicht-Lenkbarkeit von Nichtwissen}

Einleitend wird in diesem Band die These vertreten, dass Nichtwissen in aktuellen technowissenschaftlichen Diskursen eine neue und zentrale Aufmerksamkeit erhält und dass sich im Umgang damit ein Lenkungsdispositiv entwickelt. Dieses Lenkungsdispositiv zeigt übergreifend drei zentrale Strategien: das Abschätzen der Technologie, ihres Potenzials und ihrer Implikationen, das Regulieren der Technologie und ihrer potenziellen nachteiligen Implikationen auf Mensch und Umwelt und die Partizipation der involvierten Akteure und der Zivilgesellschaft in deren wissenschaftspolitischen Verhandlung.

Dabei empfehlen die meisten der im Rahmen des Abschätzungsdiskurses analysierten Publikationen, weitere und vertiefte Abschätzungen durchzuführen, den Regulierungsbedarf zu klären und die verschiedenen Anspruchsgruppen und die Zivilgesellschaft in die Entscheidungsfindung 
miteinzubeziehen. Wie diese Analyse gezeigt hat, haben diese Empfehlungen eine kaum überblickbare Masse an Abschätzungsstudien, an Regulierungsansätzen und an partizipativen Projekten produziert. Dabei sind eine Vielzahl von Berichten verfasst und zahllose Maßnahmen und Aktivitäten zur Transparenzförderung auch über elektronische Medien und Kommunikationskanäle ergriffen worden. Die verschiedenen Abschätzungsstudien zeigen indes eine hohe inhaltliche Übereinstimmung. Wie am Beispiel der Royal Society Studie gezeigt worden ist (siehe Exkurs zu Kapitel 2), beginnen solche Texte häufig mit einer Definition von Nanotechnologie, anschließend werden ihre verschiedenen Potenziale, Anwendungen und Implikationen analysiert und diskutiert und daraus relativ übergreifende Handlungsanweisungen abgeleitet. Solche Studien schließen häufig mit der Empfehlung, weitere Abschätzungen und Regulierungsanalysen vorzunehmen, die EHS-Forschung zu fördern und die beteiligten Akteure und die Zivilgesellschaft in die Entscheidungsfindung miteinzubeziehen.

Die im Regulierungsdiskurs in allen untersuchten Ländern beobachteten Ansätze, die Nanotechnologie in das bestehende Regelwerk zu integrieren, umfassen spezifische gesetzliche Maßnahmen auf der Ebene von einzelnen Anwendungen und Produkten (Nanosilber, Lebensmittel, Kosmetika), freiwillige Maßnahmen zur Gewinnung von Information und Sicherheitsdaten ebenso wie selbstregulatorische Ansätze zur Erhöhung der Labor- und der Produktsicherheit und zur Selbstdeklaration einer vorsorgeorientierten Haltung im Bereich der Nanotechnologie. Obwohl verschiedene Akteure die Überzeugung vertreten, dass die gesetzliche Regulierung einer neuen Technologie Rechtssicherheit schafft und sich so innovationsfördernd auswirkt, wird die Nanotechnologie in keinem der untersuchten Länder über die Ergänzung einzelner Vorschriften hinaus reguliert.

Stattdessen lässt sich die Delegation der Regulierung an die Gesamtgesellschaft beobachten, wie dies im Kontext des Governance-Konzepts propagiert wird (siehe Kapitel 1.1). Wie es sich hier an den verschiedenen selbstregulatorischen Verhaltenskodizes, wie dem im Exkurs zum Kapitel 3 besprochenen Nano Risk Framework gezeigt hat, lassen sich selbstregulatorische Ansätze kaum auf eine verbindliche Ebene übersetzen, auf welcher sich solche Schemen beispielsweise zu branchenüblichen Standarts entwickeln. Wie es sich bei prinzipienbasierten Verhaltenskodizes wie dem britischen ,Responsible Nanocode' gezeigt hat, unterstützt die gesellschaftliche Selbstregulierung die Tendenz, dass anstelle von übergeordneten Regulierungszielen, wie dem Schutz von Mensch und Umwelt, ver- 
stärkt anspruchsgruppenspezifische Partikularinteressen, wie Akzeptanzpflege verfolgt werden.

Der Partizipationsdiskurs umfasst einerseits die Etablierung von Anspruchsgruppendialoggremien, die ihrerseits ebenfalls Empfehlungen und Handlungsanweisungen abgeben. Darin unterscheiden sich die im Rahmen solcher Dialoggremien publizierten Berichte nicht signifikant von Abschätzungsberichten und von prinzipienbasierten Verhaltenskodizes. Dies hat sich beispielsweise an den Ergebnissen der im Exkurs zum Kapitel 4 diskutierten deutschen NanoKommission gezeigt. Die politische Umsetzung der Ergebnisse der Nanokommission ist trotz ihrer Institutionalisierung auf Regierungsebene ebenso unklar wie die in den verschiedenen Abschätzungsberichten abgegebenen Empfehlungen.

Auch die Einbindung der Zivilgesellschaft in die wissenschafts- und technikpolitische Entscheidungsfindung der Nanotechnologie gilt als schwierig. Dies ist selbst in dem im Rahmen des insbesondere in Großbritannien prominent propagierten upstream engagement bis auf die Ausnahme der partizipativen Veranstaltung des EPSRC im Rahmen der Grand Challenge Ausschreibung der Fall gewesen. Insbesondere der hohe Abstraktionsgrad der Thematik und die Problematik der politischen Umsetzung der Ergebnisse, haben zu der Schwierigkeit geführt, interessierte Bürgerinnen und Bürger für die Veranstaltungen zu gewinnen. Zudem haben solche Veranstaltungen zu eher allgemein und übergreifend formulierten Schlussberichten und Ergebnissen geführt, die sich kaum in konkretes politisches Handeln umsetzen lassen (siehe Kapitel 4.7). Wie die Analyse des Partizipationsdiskurses gezeigt hat, hat die Delegation der Entscheidungsfindung an Verfahren der Anspruchsgruppen- und der zivilgesellschaftlichen Partizipation kaum die erwünschten neuen Perspektiven im Umgang mit der Nanotechnologie gebracht.

Daraus ergibt sich die Vermutung, dass die dem Lenkungsdispositiv zugrunde liegende Annahme, dass sich das mit der Nanotechnologie verbundene Nichtwissen mit Abschätzen, Regulieren und dem Einbinden von Anspruchsgruppen und der Zivilgesellschaft erfassen und regulieren lässt, gescheitert ist. Das von verschiedenen Seiten propagierte Dispositiv, die Nanotechnologie aus unterschiedlichsten gesellschaftlichen Bereichen abzuschätzen, zu regulieren und $\mathrm{zu}$ verhandeln, hat das Nichtwissen nicht verringert. Vielmehr haben sich durch die gesellschaftliche Distribution der Expertise und der Regulierung, der Verschmelzung von entscheidungsrelevanten Wissensformen und der Auflösung von institutionenspezifischen Handlungspraktiken vormalig gesellschaftlich festgelegte Wissens- 
und Kompetenzgrenzen aufgelöst. Damit sind in Nichtwissensdiskursen sowohl die Definitions-, als auch die Entscheidungs- und die Verantwortungskompetenzen gesellschaftlich nicht mehr eindeutig zuordenbar und Fragen wie; „wer hat recht?“, „,wer entscheidet?“ und „wer trägt die Verantwortung?", sind zunehmend schwieriger zu beantworten.

Dabei scheinen sämtliche in Nichtwissensdiskursen ergriffenen Strategien im Lenken von Nichtwissen gescheitert; das Abschätzen, das Regulieren und die Partizipation. Während das Abschätzen und das Regulieren hauptsächlich an ihrer gesellschaftlichen Distribution versagen, in welcher die Zuständigkeit verwischt und die Robustheit des für die Entscheidungsfindung zu berücksichtigenden Wissens erodiert, krankt die Partizipation häufig an der Unbestimmtheit der zu verhandelnden Thematik. Dabei leidet die zivilgesellschaftliche Partizipation insbesondere am Desinteresse der Bevölkerung, abstrakte und lebensweltfremde Fragen zu verhandeln und krankt - wie auch die Anspruchsgruppenpartizipation - am häufig fehlenden Bezug zur politischen Entscheidungsfindung.

Am Fall der Nanotechnologie und der damit einhergehenden Verhandlung von Nichtwissen über potenzielle nutzbringende Anwendungen und nachteilige Implikationen ist in dieser Arbeit gezeigt worden, wie Gesellschaften der reflexiven Modernisierung in verschiedenen westlichen Industrienationen mit Nichtwissen umgehen. Daraus ergeben sich in der Abschätzung, der Regulierung und der Partizipation neu auftretende Verantwortlichkeiten und Konstellationen. Die Expertise, die in Abschätzungen und in Regulierungsentscheidungen einfließt, erfolgt nicht alleine aus den für diese Bereiche traditionellerweise zuständigen gesellschaftlichen Institutionen wie Wissenschaft und Politik. Vielmehr stammt sie auch von unterschiedlichsten gesellschaftlichen Anspruchsgruppen, wie Herstellerfirmen, Branchenverbänden, Umwelt- und Konsumentenschutzorganisationen, Stiftungen und Denkfabriken.

Damit verwischen die Grenzen zwischen unterschiedlichen entscheidungsrelevanten Wissensformen. Akademisches oder behördliches Wissen fließt nicht mehr länger alleine in die Entscheidungsfindung über Abschätzungs- und Regulierungsfragen mit ein, sondern auch gesellschaftliches Wissen, Visionen und Spekulationen (siehe Kapitel 5.1). Damit einhergehend lösen sich traditionelle, den jeweiligen gesellschaftlichen Institutionen spezifisch zuordenbare Handlungspraktiken auf. Die Wissenschaft ist auch in die Produktion von spekulativem Wissen involviert und die Politik in diejenige von wissenschaftlichem Wissen. Hersteller sind nicht nur in die Produktentwicklung involviert, sondern auch in deren Regulierung. 
Umweltorganisationen sind nicht nur an der kritischen Hinterfragung der technologischen Entwicklung, sondern ebenso an deren Regulierung und der Produktion von wissenschaftlichem Wissen beteiligt (ibid.). Dabei verwischen die traditionellen Rollen der verschiedenen gesellschaftlichen Institutionen; die Wissenschaft wird nicht mehr länger als alleinige inhaltliche Expertin aufgefasst, ebenso wie der Staat nicht mehr länger als der alleinige Träger der Regulierungsverantwortung angesehen wird.

Die vorliegende Studie hat insbesondere auch an den drei exemplarisch diskutierten Ansätzen, der Abschätzungsstudie der britischen Royal Society, dem selbstregulatorischen US-amerikanischen Nano Risk Framework von EDF und DuPont und am Stakeholerpartizipationsgremium der deutschen NanoKommission gezeigt, wie diese ins Leere laufen. Alle drei Ansätze geben Empfehlungen ab und Handlungsanweisungen vor. Es zeigt sich jedoch, dass sich kaum jemand dazu verpflichtet fühlt, diese auch umzusetzen. Die britische Regierung ist für die mangelnde Umsetzung der Empfehlungen der RS\&RAE breit kritisiert worden (siehe Kapitel 2.5.3). Die von EDF und DuPont angestrebte Übernahme ihres Nano Risk Frameworks durch andere Firmen und dessen Etablierung als Referenzschema hat sich nicht erfüllt (siehe Kapitel 3.3.4). Ebenso wenig sind die fünf Prinzipien der NanoKommission von den angesprochenen Anspruchsgruppen aufgenommen worden. Hier haben sich teilweise nicht einmal die in der Nanokommission vertretenen Akteure offiziell dazu bekannt (siehe Kapitel 4.6.1). Darin liegt die zentrale Problematik der Lenkungsfragen in Nichtwissensdiskursen: Sämtliche betroffenen Akteure machen im Diskurs mit, sie kooperieren und verhandeln, aber niemand trifft Entscheidungen, niemand setzt sie um und niemand trägt die Verantwortung.

Durch die Verwischung dieser vielfältigen Grenzen wird nun alles möglich und es entsteht eine Situation maximaler Gestaltungsoffenheit. Aus Visionen werden neue Technologien generiert und die traditionell für deren Abschätzung und Regulierung zuständigen Institutionen verlieren durch die gesellschaftliche Distribution der Expertise und der Regulierungsverantwortung an Bedeutung. Die Entscheidungs- und Strategiefindung in Abschätzungs-, Regulierungs- und Partizipationsdiskursen wird so ad absurdum geführt. Dadurch läuft das Lenkungsdispositiv ins Leere. Seine Stoßrichtung, Nichtwissen definierbar, kalkulierbar und regulierbar zu machen scheitert. Das Nichtwissen nimmt zu und bleibt trotz der intensiven gesamtgesellschaftlichen Bestrebungen weder lenkbar noch fassbar. Damit zeichnen sich Nichtwissensdiskurse durch einen Zustand maximaler Gestaltungsoffenheit und minimaler Verbindlichkeit aus. 